\title{
La Réplica en el Análisis Económico Aplicado
}

\author{
ANASTASIA HERNÁNDEZ AlEMÁN ${ }^{\text {a }}$, CARMELO J. LEÓN ${ }^{\text {a }}$ \\ a University Institute of Tourism and Sustainable Economic Development. Las Palmas de Gran \\ Canaria University, Campus Universitario de Tafira, 35017 Las Palmas de Gran Canaria, Spain. E- \\ mail: anastasia.hernandez@ulpgc.es, cleon@daea.ulpgc.es
}

\section{RESUMEN}

Este artículo induce a la reflexión acerca de la economía como ciencia. Uno de los principios básicos del método científico es la "reproductibilidad", esto es, la capacidad para repetir un experimento. En ello se fundamenta la publicidad de sus resultados y su potencial verificación por la comunidad científica. Este pilar básico del método científico parece estar en cuestión en el análisis económico. Así surgen en la literatura, más recientemente, numerosos artículos que discuten la necesidad de impulsar y promover la réplica de los estudios empíricos aportando lo datos y los códigos que faciliten esta tarea a los investigadores. Ello como parte esencial del progreso científico, aportanto robustez y transparencia a los resultados ya obtenidos y publicados.

Palabras clave: Método científico, economía y réplica.

\section{Replication in Applied Economic Analysis}

\begin{abstract}
This paper leads to deliberate on economics as a science. One of the basic principles of the scientific method is the "reproducibility", so an experiment can be tested o repeat it. This is based on the publicity of its results and its potential verification by the scientific community. Nowadays, this basic rule of the scientific method seems to be in question in the economic analysis. Then, we find in the literature, more recently, numerous articles that discuss the need to impulse and promote the replication of empirical studies. Researchers should obtain the author's dataset and codes that facilitate the replication or verification or reproducibility. This is an essential part of scientific progress, providing robustness and transparency to the results already obtained and published.
\end{abstract}

Keywords: Scientific Method, Economy and Replication.

Clasificación JEL: B4, C5

Artículo recibido en octubre de 2017 y aceptado en diciembre de 2017

Artículo disponible en versión electrónica en la página www.revista-eea.net, ref. ə-36114 


\section{INTRODUCCIÓN}

En los últimos años, la investigación en economía aplicada, en particular, se viene haciendo eco de la necesidad de promover e incentivar "la réplica"; como así se viene haciendo en otros ámbitos de la ciencia, como por ejemplo, en ciencias de la salud. Se pone en evidencia que el progreso de la ciencia, al tiempo que su credibilidad depende en parte de que sea posible la réplica así como su divulgación, algo que en el ámbito de la economía no resulta muy frecuente como veremos a lo largo de este artículo.

En la práctica hay un déficit de estudios que sean réplica de otros ya publicados. Circunstancia que también se observa en España. Surge aquí la necesidad, en primer lugar, de profundizar en el concepto de "réplica". Conforme a la definición de la Real Academia Española de la Lengua, la "réplica" es la copia exacta de algo, especialmente de una obra artística. En el caso que nos ocupa sería algo así como la "reproducción" de unos resultados ya obtenidos. También se hace acopio de otros términos como "repetición,...,"; acción de replicar, y más concretamente referido al ámbito del derecho: "segundo escrito del actor en el juicio de mayor cuantía para impugnar la contestación y la reconvención, si la hubo, y fijar los puntos litigiosos”.

Así, en un sentido estricto en el ámbito de la economía, la réplica sería la "pura" reproducción o repetición de un trabajo anterior, duplicación de los resultados publicados (mismo método, misma muestra y población) frente a lo que se asimilaría siguiendo a Hamermesh (2007) a una "statistical replication". Esto es, replicar un análisis con diferente muestra, misma población e idéntico modelo o especificación. Por último, la "scientific replication" consistiría en emplear diferente muestra y población, método similar pero no idéntico. Clemens (2017) separa replicación de robustez, estableciendo una diferencia entre ambos términos. Así, para la "verificación" (réplica) se usaría la misma muestra, población y misma especificación empírica. Para la "reproducción” (réplica) se emplearía diferente muestra, misma población y misma especificación. Mientras que la "robustez o reanálisis" emplearía la misma muestra y población pero diferente especificación. Por último la "extensión” (robustez) usaría diferente muestra y población pero la misma especificación. La "pura replicación” de Hamermesh coincide con la "verificación" de Clemens. La "statistical replication" de Hamermesh se corresponde con la "reproducción" de Clemens; mientras que la "scientific replication" de Hamermesh entra en el campo de la robustez de Clemens.

Lo más frecuente en el análisis económico es la "scientific replication" o "replication with extension" consistente en plantear un modelo diferente probablemente más sofisticado en la línea de las nuevas aportaciones metodológicas o ajustar el modelo con interacciones entre variables diferentes, otra muestra, y muy probablemente, diferente enfoque pero similar (De Jesús 
Romo, 2016). No obstante, como afirma McCullough et al. (2006) la economía no ha adoptado plenamente el método científico por cuanto no hay tradición en la práctica de la réplica. Pocas son las revistas que exigen aportar los datos que permitan replicar el modelo, y aún cuando se piden, no siempre son aportados impunemente por los autores de los trabajos. Por otra parte, son pocas las revistas dispuestas a publicar una réplica si quiera esté en relación con un trabajo publicado anteriormente, salvo que fuera para reforzar los resultados ya publicados anteriormente. Por ejemplo, Labour Economics considera ocasionalmente la revisión o reproducción ("review articles") pero no la réplica ("pura" reproducción). La Journal of Political Economy contempla expresamente la réplica de forma que se deben aportar los datos empleados en el análisis. En la misma línea se contempla en la prestigiosa American Economic Review que lleva publicados como réplica el 29\% de los artículos, siendo una minoría frente al 59\% que son réplica o extensión (Berry et al., 2017). La Journal of Human Resources regula expresamente las condiciones para realizar una réplica así como también la puesta a disposición de los datos y los códigos de programación empleados en el análisis por parte de los autores de los manuscritos aceptados. Se puede otorgar una excepción a los criterios generales establecidos por la revista para la réplica cuando se cumplan los siguientes requisitos: 1) existe algún método por el cual otros investigadores pueden obtener los datos, y 2) los autores se comprometen a proporcionar orientación sobre la obtención de los datos. Al solicitar una exención, se debe aportar una breve explicación de por qué se requiere la exención y cómo se cumple con estos criterios. Journal of Applied Econometrics introduce la réplica en el año 2003 con Badi H. Baltagi que se dedicó casi en exclusiva a la cuestión de la reproducción de los resultados empíricos publicados en esta revista. Actualmente la réplica en esta revista se ha extendido también a los resultados publicados en otras revistas como son: Econometrica, American Economic Review, Journal of Political Economy, Quarterly Journal of Economics, Review of Economics and Statistics, Review of Economic Studies, Journal of Econometrics, Journal of Business and Economic Statistics y Economic Journal. Se regulan expresamente los criterios para la réplica, distinguiendo ésta según sea una réplica en sentido estricto, o bien, una réplica en sentido amplio (extensión). En la Tabla 1 se muestran algunas de las revistas que publican trabajos que son réplica o reproducción de otros estudios publicados anteriormente.

Tabla 1

Revistas que publican la réplica y la reproducción o revisión

\begin{tabular}{|l|l|}
\hline American Economic Review & Economic Development and Cultural Change \\
\hline Econ Journal Watch & Experimental Economics \\
\hline Journal of Human Resources & Explorations in Economic History \\
\hline Economics of Education Review & International Journal of Forecasting \\
\hline Empirical Economics & Journal of Economics and Statistics \\
\hline
\end{tabular}


Tabla 1 (continuación)

Revistas que publican la réplica y la reproducción o revisión

\begin{tabular}{|l|l|}
\hline Journal of Applied Econometrics & Review of International Organizations \\
\hline Journal of Political Economy & Labour Economics \\
\hline
\end{tabular}

Fuente: Duvendack et al. (2015:175). Elaboración propia.

En la Tabla 2 se observan los porcentajes que representan las réplicas publicadas según la revista. Journal of Applied Econometrics publica un mayor volumen de artículos que son réplica de otros publicados anteriormente. Le siguen Journal of Human Resources y American Economic Review. No obstante, y a pesar de estos esfuerzos, la réplica así como la reproducción no se prodigan profusamente en el ámbito de la economía. En este sentido, Hamermesh (2017) señala que se hacen muy pocas réplicas en sentido puro (Hamermesh, 2007b); se hace poca "ciencia dura" en economía, cuestionándose las razones de este escaso mercado. Tal vez la explicación se halla en que hay bienes sustitutivos de las réplicas, falta de incentivos o la propia imposibilidad de llevar a cabo la réplica. En el ámbito de la economía del trabajo, la probabilidad de que un artículo citado sea similar o repita explícitamente el trabajo original disminuye a lo largo de los años después de la publicación original, y lo hace linealmente. La escasez de réplicas es aún mayor, respondiendo a una función cuadrática, en aquéllos artículos clasificados como "inspirados metodológicamente" en el estudio original. Por contra, el 92,8\% de los 111 artículos que son réplica de otros publicados son clasificados en la categoría "related to"; esto es, meramente relacionados con el original. Apenas se encontraron réplicas clasificadas como "similar" o "réplica directa" del estudio original. Los artículos publicados en las revistas más prestigiosas "no pueden ser" fácilmente refutados por réplicas que pongan en cuestión esos resultados.

Tabla 2

Distribución de las réplicas según la revista

\begin{tabular}{|l|c|c|}
\hline \multicolumn{1}{|c|}{ Revista } & $\begin{array}{c}\text { Frecuencia } \\
\text { \% } \\
\left(\mathbf{n}^{\circ} \mathbf{2} \mathbf{2 0 1 5}\right.\end{array}$ & $\begin{array}{c}\text { Frecuencia } \\
\mathbf{\%} \\
\left(\mathbf{n}^{\mathbf{0}} \mathbf{-} \mathbf{2 0 1 7}\right.\end{array}$ \\
\hline 1. Journal of Applied Econometrics & $19,1(31)$ & $19,7(46)$ \\
\hline 2. Journal of Human Resources & $11,7(19)$ & $8,5(20)$ \\
\hline 3. American Economic Review & $9,3(15)$ & $12(28)$ \\
\hline 4. Econ Journal Watch & $6,8(11)$ & $6(14)$ \\
\hline 5. Journal of Development Studies & $6,2(10)$ & $4,3(10)$ \\
\hline 6. Experimental Economics & $5,6(9)$ & $3,8(9)$ \\
\hline 7. Applied Economics & $4,3(7)$ & $3,4(8)$ \\
\hline 8. Empirical Economics & $4,3(7)$ & $3,8(9)$ \\
\hline 9. Journal of Economic and Social Measurement & $3,7(6)$ & $3,0(7)$ \\
\hline 10. Public Choice & $3,7(6)$ & $2,6(6)$ \\
\hline 11. Journal of Political Economy & $1,9(3)$ & $3,4(8)$ \\
\hline
\end{tabular}


Tabla 2 (continuación)

Distribución de las réplicas según la revista

\begin{tabular}{|c|c|c|}
\hline Revista & $\begin{array}{c}\text { Frecuencia } \\
\% \\
\left(n^{\circ}\right) \_2015\end{array}$ & $\begin{array}{c}\text { Frecuencia } \\
\% \\
\left(n^{\circ}\right)^{-} \quad 2017\end{array}$ \\
\hline 12. Labour Economics & $1,9(3)$ & $1,3(3)$ \\
\hline 13. Economic Inquiry & $1,2(2)$ & $1,7(4)$ \\
\hline $\begin{array}{l}\text { 14. Journal of Environmental Economics and } \\
\text { Management }\end{array}$ & $1,2(2)$ & $0,6(2)$ \\
\hline 15. Quarterly Journal of Economics & $1,2(2)$ & $1,3(3)$ \\
\hline 16. Economics Bulletin & $0,6(1)$ & $1,3(3)$ \\
\hline 17. Review of International Organizations & $1,2(2)$ & $0,6(2)$ \\
\hline 18. American Economic Journal: Applied Economics & $0,6(1)$ & $0,2(1)$ \\
\hline 19. Public Finance Review & $0,6(1)$ & $4,3(10)$ \\
\hline 20. American Law and Economics Review & $0,6(1)$ & - \\
\hline 21. Applied Financial Economics & $0,6(1)$ & - \\
\hline 22. Conflict Management and Peace Science & $0,6(1)$ & - \\
\hline 23. Econometrica & $0,6(1)$ & - \\
\hline 24. Economic Journal & $0,6(1)$ & - \\
\hline 25. European Economic Review & $0,6(1)$ & - \\
\hline 26. Health Economics & $0,6(1)$ & - \\
\hline 27. International Economics and Economic Policy & $0,6(1)$ & - \\
\hline 28. International Review of Applied Economics & $0,6(1)$ & - \\
\hline 29. Journal of Development Economics & $0,6(1)$ & - \\
\hline 30. Journal of Development Effectiveness & $0,6(1)$ & - \\
\hline 31. Journal of International Development & $0,6(1)$ & - \\
\hline $\begin{array}{l}\text { 32. Journal of International Trade \& Economic } \\
\text { Development }\end{array}$ & $0,6(1)$ & - \\
\hline 33. Journal of Law and Economics & $0,6(1)$ & - \\
\hline 34. Journal of Money, Credit, and Banking & $0,6(1)$ & - \\
\hline 35. Journal of the European Economic Association & $0,6(1)$ & - \\
\hline 36. Journal of Urban Economics & $0,6(1)$ & - \\
\hline 37. Marketing Letters & $0,6(1)$ & - \\
\hline 38. Proceedings of the National Academy of Sciences & $0,6(1)$ & - \\
\hline 39. Quarterly Journal of Business and Economics & $0,6(1)$ & - \\
\hline 40. Review of Austrian Economics & $0,6(1)$ & - \\
\hline 41. Review of Economics and Statistics & $0,6(1)$ & - \\
\hline 42. Review of Financial Studies & $0,6(1)$ & - \\
\hline 43. Social Science \& Medicine & $0,6(1)$ & - \\
\hline 44. World Development & $0,6(1)$ & - \\
\hline
\end{tabular}

Fuente: Duvendack et al. (2015; 2017). Elaboración propia ${ }^{1}$.

\section{En el último año ${ }^{2}$, la Journal of Applied Econometrics ha publicado un total} de 65 artículos originales y 13 artículos que son réplica de otros artículos ya

${ }^{1}$ Para llevar a cabo la actualización de la tabla en cuanto a la réplica según la revista, se ha acudido a la web de cada revista a efectos de contrastar los artículos originales de aquellos que son réplica de otros originales publicados anteriormente. También se ha consultado la web The Replication Network. 
publicados, lo que representa el $16,67 \%$ con respecto al total de artículos publicados y el $20 \%$ con respecto al total de artículos originales. Así pues, parece mantener una tendencia creciente en cuanto a la ratio de réplicas publicadas. Las áreas replicadas tienen que ver con la economía del bienestar y la renta, el crecimiento económico y los aspectos metodológicos (e.g. logit mixto, regresión de cuantiles incondicional, métodos de control sintéticos).

Sukhtankar (2017) hace una revisión de la réplica en el ámbito de la economía del desarrollo durante el periodo de 2000 a 2015 ambos incluidos en una de las cinco primeras revistas de economía de interés general (o de las cinco más próximas) ${ }^{3}$. Haciendo una búsqueda en Econlit por artículo publicado analiza el tipo de réplica y su correlación con el artículo replicado. Resalta lo interesante de los tópicos replicados, el incremento considerable de los artículos empíricos publicados en el área de desarrollo como consecuencia de la mejora de la metodología, la disponibilidad de datos y la proliferación de ensayos de control aleatorio (RCTs). De los 1.390 artículos, el 73,23\% son artículos empíricos que no se enmarcan en ensayos aleatorios, el 18,13\% son artículos teóricos y el restante 8,64\% se enmarcan en ensayos o pruebas de control aleatorio (RCTs). De los 1.138 artículos empíricos, sólo 71 que representan el 6,2\% fueron replicados, bien en un artículo, bien en un documento de trabajo. Este número desciende a 37 $(3,3 \%)$ si sólo se consideran los que fueron publicados. Siguiendo la clasificación de Clemens (2017) respecto a la réplica, el 32,4\% de los artículos publicados y replicados lo fueron en la categoría de "verificación"; el porcentaje restante se enmarca en el análisis de la robustez bien sea re-análisis, bien una extensión. Así pues, la gran mayoría de las réplicas de robustez plantean una especificación econométrica diferente, o bien, diferente muestra de datos. Teniendo en cuenta los incentivos a la publicación, los autores sólo citan su artículo como réplica cuando ésta lo es en un sentido estadísticamente estricto respecto de otro artículo ya publicado, y no cuando lo es en un sentido más amplio o extenso. Sukhtankar (opus cit.,) también encontró que los artículos replicados son citados una media de cuatro veces más que los que no lo son.

Algunos de los beneficios más inmediatos de la réplica o de la reproducción derivan del ahorro que supone aprovechar los códigos que ya han sido escritos para la estimación de los modelos, los datos que ya han sido recogidos y tratados, etcétera. Revisando y verificando toda esta información, algo que los referees no hacen, se aseguran unos resultados ya publicados que podrán ser archivados. El ejemplo de McCullough (2006) es muy ilustrador de los efectos positivos de esta práctica. El modelo de Hansen, Heaton y Ogaki (1988) fue

2017.

${ }^{3}$ American Economic Journal: Applied Economics (AEJAE), American Economic Journal: Economic Policy (AEJEP), the Economic Journal (EJ), the Journal of the European Economic Association (JEEA) y Review of Economics and Statistics (ReStat). 
usado en varias ocasiones adaptado según las necesidades. Ogaki publicó los códigos por lo que la adaptación resultó más sencilla. Hamilton proporcionó el código de Gauss para que todos lo usaran y de ello ha derivado toda una industria. Lo más significativo a destacar es el tiempo que se ahorraría a estudiantes e investigadores si se facilitaran los datos y los códigos. Facilitaría la verificación de los resultados obtenidos, se profundizaría en el manejo de las herramientas metodológicas, se compararían resultados al tiempo que se podría aportar nuevo conocimiento. Existe toda una motivación para movernos en esta dirección. No obstante, la réplica suele ser vista en la mayoría de las ocasiones como un ataque a un trabajo previo, y en otras ocasiones, como la búsqueda de notoriedad del "investigador principiante", cuestionando los resultados del "investigador senior”. En este sentido, forma parte de una buena investigación reconocer con humildad cuando se ha cometido un error y agradecer a quiénes se tomaron la molestia y dedicaron su tiempo a hacer la replicar y encontrar el error. Lo determinante desde el punto de vista científico es que no se propague un error.

El objetivo de este trabajo es incitar a la reflexión sobre la réplica en economía, su impacto en la ciencia económica, así como, la necesidad de promover la publicación de trabajos que sean réplica de otros anteriores como mayor enriquecimiento y transparencia en el ámbito objeto de análisis, y también en el ámbito educativo o formativo. El resto del artículo se organiza como sigue. En el siguiente apartado se analizan algunas de las réplicas publicadas en economía en los últimos años. En el tercer apartado se analizan tres ejemplos de artículos de economía publicados en revistas de prestigio, según el grado de dificultad que podría conllevar la réplica. En el cuarto, y último apartado, se resumen las conclusiones.

\section{UNA RÉPLICA}

Para completar esta discusión, y sin profundizar en los asuntos que son objeto de análisis, se aportan en este apartado como ejemplo algunas de las réplicas realizadas en el pasado, y también, algunas de las más recientes. Mercer y Reed (2015) replicaron el estudio de Toya y Skidmore (2007) no encontrando evidencia significativa estadísticamente y robusta con respecto a la relación causal entre el nivel de desarrollo económico de un país y las consecuencias económicas de un desastre natural. Tomando como base del análisis de réplica la base de datos original, y actualizando las variables a un periodo más reciente, MR replican los test de TS observando que a excepción de un sólo caso, las variables renta, nivel educativo, tamaño del gobierno, apertura económica y nivel de desarrollo del sistema financiero no obedecen a una relación causal estadísticamente significativa como factores determinantes de un menor número de muertes y daño causado por un desastre natural. La excepción consiste en que MR encuentran una asociación entre un nivel de renta elevado y menores pérdidas económicas. El 
artículo hasta el momento de la réplica había sido citado cuarenta veces en la web de Science y alrededor de 180 veces en Google Scholar. Se discute que, si bien los países más desarrollados o más ricos pueden mejorar sus infraestructuras y hacerlas más resistentes a un desastre natural minimizando con ello los daños, cómo es posible que ello no afecte a las fatalidades?. La razón que aporta el análisis de $\mathrm{MR}^{4}$ es que los resultados de TS adolecen de colinealidad entre algunas variables y hay ausencia de robustez que permita garantizar una relación causal estadísticamente significativa entre las variables. Esto no quiere decir que la relación entre ambas circunstancias no exista en la realidad. A su vez Toya y Skidmore (2015) hicieron una réplica de la réplica centrando el problema en aspectos metodológicos (censura de los datos, regresión a intervalos) y respecto a los datos (pooled, datos de panel equilibrado o desequilibrado). Así pues, la réplica vino a profundizar y ampliar el análisis original en varias dimensiones, añadiendo una importante contribución al análisis empírico en el ámbito de la economía. Liebowitz (2017) replica cuatro “cuasi-experimentos" usando idénticos datos y métodos estadísticos, cuando es posible. El estudio concluye que los cuatro experimentos llevados a cabo por Oberholzer-Gee y Strumpf publicados en Journal of Political Economy en 2007 contienen errores importantes e inconsistencias que llevan en algún caso a un cambio en las conclusiones en términos cualitativos, y en otros, a cuestionar la significación estadística de los resultados por insuficiencia de observaciones.

Sheldon (2017) replica las estimaciones de Holtz-Eakin y Selden (1995) cuyos resultados se publicaron en Journal of Political Economy. Los resultados del trabajo de HE-S se sostienen sorprendentemente bien, ampliando la muestra a 20 años y 45 países. Sólo se advierten unas pequeñas diferencias. El análisis se enmarca en relación con la Curva de Kuznets propuesta a principios de 1990 aplicada al ámbito ambiental de forma que a medida que aumenta el ingreso per cápita de un país partiendo de niveles muy bajos, la contaminación primero empeora hasta alcanzar un umbral de renta per cápita, mejorando a partir de este punto la calidad ambiental a causa de las ganancias generadas en este proceso. Esta relación adoptaría la forma de U invertida. HE-S encuentran evidencia que sustenta la forma de la curva en U invertida entre emisiones de carbono y el ingreso per cápita pero observan que el punto de inflexión era elevado en relación con los niveles de ingresos actuales. De este modo pronostican que la mayoría de los países continuarán operando en la parte ascendente de la curva hasta 2100 lo que se traduce en un aumento constante de las emisiones. Sheldon (opus cit.,) obtiene, en primer lugar, un punto de inflexión

\footnotetext{
${ }^{4}$ Emplean para el análisis una metodología más sofisticada. Si bien TS emplean un pooled de datos estimado por mínimos cuadrados ordinarios, MR tienen en cuenta el truncamiento o censura de los datos, la falta de simetría en los datos sobre desastres naturales, los efectos temporales y espaciales y una estructura de errores más flexible.
} 
que está por debajo de las estimaciones originales. En segundo lugar, las emisiones en 2100 pueden disminuir en la última parte del siglo dependiendo del crecimiento de la población y de la convergencia global. Por último, las predicciones a corto y medio plazo son mayores que las estimaciones originales las cuales parecen ser muy optimistas.

Los criterios de selección de un artículo o varios a efectos de llevar a cabo la réplica pueden obedecer a diferentes finalidades. Entre los criterios más frecuentes que motivan la réplica cabe mencionar los siguientes:

- verificación de los resultados más relevantes a efectos de extender la muestra a otras aplicaciones, países, periodo temporal, como hacen por ejemplo, Mercer y Reed (2015) que actualizan la base de datos original a un periodo más reciente; por su parte, Sheldon (2017) amplía la base de datos temporal y espacialmente,

- validar los resultados en términos cualitativos y su interpretación, como por ejemplo, estudia Liebowitz (2017) en la réplica de los cuatro experimentos,

- probar la robustez de la metodología empleada en el análisis, proponiendo alternativas, como por ejemplo, en Toya y Skidmore (2015) cuando hicieron una réplica de la réplica desde el punto de vista metodológico.

La web "Replications in Economics" (wiki) que se comentará en el siguiente apartado cuenta con un sistema de votaciones respecto de aquellos estudios que se consideran más relevantes a efectos de réplica. No obstante, este procedimiento no cuenta con suficiente éxito por cuanto apenas hay votaciones. Por otra parte, existe toda una literatura en el ámbito de la economía de la educación que analiza el procedimiento a seguir en economía para que un artículo sea replicable (Ball y Medeiros, 2012).

\section{DATOS}

Los datos disponibles evidencian no sólo la escasez de posibilidades existentes para la réplica a la vista de las pocas revistas que facilitan esta tarea ${ }^{5}$; sino que además, se deben tener en cuenta las complicaciones que surgen cuando se esboza alguna posibilidad para la réplica, como en los ejemplos que se muestran a continuación. En varias webs como, por ejemplo, en la American Economic Association $^{6}$ se pueden consultar algunos de los artículos publicados según la revista, y en algunos casos, se pueden acceder a los datos y a los códigos de programación de los modelos estimados. Otras webs como The Replication

\footnotetext{
${ }^{5}$ Duvendack et al. (2017) encuentran que sólo 28 de las 333 revistas de economía consultadas publican regularmente los datos y los códigos. Regularmente implica, al menos, el 50\% de los artículos empíricos publicados recientemente.

${ }^{6}$ https://www.aeaweb.org
} 
Network $^{7}$ o Research Papers in Economics ${ }^{8}$ también ofrecen la posibilidad de localizar estudios replicados y publicados en economía en diferentes áreas de especialización. No obstante, pocas veces ocurre que coincidan las tres posibilidades: tener acceso al artículo, a los datos y a los códigos de programación. Aún cuando se den todas las coincidencias puede suceder que los datos estén en una determinada versión de un determinado software y su descarga no sea posible, bien por no disponer de este programa informático, bien por no disponer de la versión. En los ejemplos que se presentan a continuación los cuales han sido seleccionados según el grado de dificultad para poder realizar la réplica se ha optado por acudir a la web of Science de la Universidad de Gottinger "Replications in Economics" (wiki ${ }^{9}$ ) que ofrece un amplio abanico de posibilidades para replicar un artículo de entre un total de 2.108 estudios publicados que no cuentan con réplica. En esta web se proporciona una ficha por artículo publicado en la que consta si los datos están disponibles, si es posible acceder a los códigos de programación y al archivo de lectura. Así pues, se procede a la selección de tres artículos a efectos de comprobar las posibilidades de réplica que cada uno de ellos ofrece:

Tabla 3

La réplica según el grado de dificultad

\begin{tabular}{|c|c|c|c|c|c|c|}
\hline Autores & Título & Revista & $\begin{array}{l}\text { Códigos de } \\
\text { programación }\end{array}$ & $\begin{array}{c}\text { Base de } \\
\text { datos }\end{array}$ & Apéndice & $\begin{array}{c}\text { Programa } \\
\text { informático }\end{array}$ \\
\hline $\begin{array}{l}\text { Alex Bryson, G. } \\
\text { Mackerron (2017) }\end{array}$ & $\begin{array}{c}\text { Are you happy } \\
\text { while you work? }\end{array}$ & $\begin{array}{c}\text { The Economic } \\
\text { Journal, } \\
127: 165-125\end{array}$ & No disponible & $\begin{array}{c}\text { No } \\
\text { disponible }\end{array}$ & $\begin{array}{c}\text { No } \\
\text { disponible }\end{array}$ & - \\
\hline $\begin{array}{l}\text { David Seim } \\
\quad(2017)\end{array}$ & $\begin{array}{l}\text { Behavioral } \\
\text { responses to } \\
\text { wealth taxes: } \\
\text { evidence from } \\
\text { Sweden }\end{array}$ & $\begin{array}{l}\text { American } \\
\text { Economic } \\
\text { Journal: } \\
\text { Economic } \\
\text { Policy, } 9 \text { (4): } \\
\text { 395-421 }\end{array}$ & Disponible & $\begin{array}{c}\text { No } \\
\text { disponible }\end{array}$ & Disponible & Stata \\
\hline $\begin{array}{c}\text { Stephen P. } \\
\text { Holland, Erin T. } \\
\text { Mansur, Nicholas } \\
\text { Z. Muller, Andrew } \\
\text { J. Yates } \\
\text { (2016) }\end{array}$ & $\begin{array}{l}\text { Are there } \\
\text { environmental } \\
\text { benefits from } \\
\text { driving electric } \\
\text { vehicles? The } \\
\text { importance of } \\
\text { local factors }\end{array}$ & $\begin{array}{l}\text { American } \\
\text { Economic } \\
\text { Review, } 106 \\
\text { (12): } 3700- \\
3729\end{array}$ & Disponible & Disponible & Disponible & Stata 13 \\
\hline
\end{tabular}

Fuente: Elaboración propia.

El primer artículo de la Tabla 3, "Are you happy while you work? es un ejemplo de artículo de imposible réplica. Los datos no están disponibles, los

\footnotetext{
${ }^{7}$ https://replicationnetwork.com/replication-studies/

${ }^{8}$ http://repec.org/

${ }^{9}$ http://replication.uni-goettingen.de/wiki/index.php/Category:Study_lacking_replication
} 
códigos tampoco lo están y se desconoce el programa informático empleado para el análisis. Este tipo de inconvenientes constituye una de las primeras barreras, y la más frecuente, a superar cuando un investigador se dispone a replicar un artículo previamente publicado. Los datos proceden de una app gratuita conocida como Mappiness ${ }^{10}$ descargable en el iPhone.

El segundo ejemplo que se aporta en la Tabla 3, "Behavioral responses to wealth taxes: evidence from Sweden" es un ejemplo de artículo cuya réplica aparentemente es factible. No obstante, los inconvenientes con respecto a los datos suponen una dificultad que prácticamente la hacen imposible en un sentido estricto ("puro"). Los datos no están disponibles online pero puede ser solicitados al servicio estadístico de Suecia. No hay una descripción adecuada de los microdatos que se emplean para obtener el ahorro privado con lo cual tampoco es muy factible saber con exactitud cuál es la base estadística empleada denominada como "KU31". No está claro si se emplea la estadística de ingresos de los hogares, o bien, la estadística financiera (depósitos netos) de los hogares, u otras que cuentan entre sus variables con el ahorro de los hogares. Se sabe que los microdatos deben contar con el número de la seguridad social para poder hacer el "merge" entre los diferentes archivos de diferentes estadísticas pero se desconoce con exactitud la operación estadística empleada. Una vez consultados los códigos de programación tampoco es posible deducir esta cuestión, por lo que inevitablemente éste es un caso que requerirá de la colaboración del autor del artículo para poder llevar a cabo la réplica.

El tercer ejemplo que se propone, "Are there environmental benefits from driving electric vehicles? The importance of local factors" cumple todos los requisitos para poder llevar a cabo la réplica. El artículo es de acceso libre en la revista American Economic Review por lo que es posible su descarga online. Igualmente es posible descargar la bases de datos con los códigos de programación (do_file) y el $p d f$ de lectura de los archivos. El único inconveniente en este caso es que los datos están en formato Stata 13 por lo que para su lectura se requiere este programa informático y esta versión; o bien, una versión anterior siempre y cuando sea posible guardar estos datos en esa versión previa con el comando "saveold filename, versión (12)"; o bien, disponer de una versión posterior en la que también es posible su lectura.

Sin duda, la variedad de programas informáticos existentes en el mercado, sus diferentes versiones, así como las propias limitaciones entre versiones, es otro inconveniente cuando se aborda la réplica. En este sentido, estas limitaciones se podrían solventar transformando los datos en formato $t x t$ con su correspondiente archivo de lectura de forma que pudiera ser leído en cualquier programa estadístico con independencia de la versión. La siguiente cuestión que se nos

${ }^{10}$ http://www.mappiness.org.uk/ 
plantea es si la programación optimiza el tiempo de estimación de los resultados. Otro tipo de barreras además de las comentadas anteriormente derivan del poco interés que presentan las revistas científicas a publicar réplicas. Primero, porque no se trata de artículos originales, y segundo, porque se pone en cuestión la intencionalidad ${ }^{11}$ de la réplica, y con ello, las consecuencias que pudiera derivar en cuanto a la frecuencia con la que el artículo de réplica pudiera ser citado. Más de la mitad de las revistas de la Tabla 1 sólo ha publicado una réplica, dos como máximo, a lo largo de toda su trayectoria. Ello teniendo en cuenta además que todas han publicado, al menos, en una ocasión, una réplica. El 75\% de las réplicas publicadas que recoge la Tabla 1 se concentran en diez revistas. Se trata de revistas que llevan publicadas más de tres réplicas. No parece que haya mucho incentivo como para dedicar tiempo y esfuerzo a la réplica a pesar de las enormes ventajas, credibilidad y transparencia que aportaría a la economía como ciencia social. Un artículo que se replica y obtiene iguales resultados que el publicado originalmente adquiere mayor fuerza y robustez que sin la existencia de la réplica.

\section{CONCLUSIONES}

En los últimos años se ha realizado un gran esfuerzo por parte de las revistas de economía más prestigiosas en promover la réplica, facilitando el acceso a los códigos y a los datos de los artículos empíricos publicados. Hay equipos de investigación como Camerer et al. (2016) que han replicado 18 estudios en economía experimental. Parece claro que la reproducción de los resultados en análisis económico aplicado se hace necesario particularmente en el ámbito educativo pero además sería un buen ejercicio ahondar en la réplica pues induciría a la reflexión, al contraste y al desarrollo de esta ciencia social. No obstante, ante la dificultad de llevar a cabo la réplica en numerosos artículos cabe plantearse si los experimentos en economía son replicables. Chang y Li (2015) sólo pudieron replicar 22 artículos siguiendo las instrucciones de los archivos de lectura de los autores, respecto de los 67 artículos seleccionados en base a dos criterios y publicados en 13 revistas. El principal inconveniente para no replicar los restantes 45 artículos fue la no disponibilidad de los datos y los códigos por parte de los autores. Tal y como se ha comprobado en el apartado anterior de este artículo, este es el principal inconveniente pero no el único porque aún contando con los códigos y los datos necesitamos disponer del software adecuado. También se ha visto en el apartado anterior que en ocasiones se emplean datos confidenciales o datos que no están disponibles en la web como en el caso del primer artículo cuyos datos procedían de una app libre, por lo que en estos casos no es posible la réplica en un sentido puro.

${ }^{11}$ En ocasiones, la réplica es vista con intenciones malévolas, de bullying o persecución respecto de los autores del artículo originario. 
Como conclusión se observa que a pesar de las dificultades, en ocasiones la réplica en sentido estricto es posible, y cuando no es posible, en la mayoría de los casos se debe a dificultades materiales que son subsanables, como son la disponibilidad de los códigos y los datos en un formato básico que permitan ser leídos en cualquier software estadístico. Es claro que cuando se trata de datos confidenciales no es posible la réplica, debiendo creernos lo que se publica.

Tal vez una reflexión en las editoriales acerca de impulsar una revista, línea o sección que tuviera por objetivo sólo la publicación de réplicas tanto en un sentido amplio inspiradas en metodología, resultados o ideas procedentes de otros trabajos ya publicados, como en un sentido más estricto, como pura reproducción de trabajos ya publicados animaría a estudiantes e investigadores a profundizar en un determinado ámbito de la economía, además de proporcionar una mayor confianza y transparencia en los resultados ya publicados.

La réplica en positivo reforzará los resultados obtenidos y publicados y la réplica en negativo purifica los errores. El depósito de los datos salvo las bases de datos privadas así como los códigos empleados para la estimación de los modelos constituiría un estímulo para nuevos investigadores, formadores (investigador senior) y un aliciente para impulsar nuevas líneas de investigación. Desde esta perspectiva las ventajas superan a los riesgos que conlleva esta iniciativa. Cabe cuestionarnos finalmente si los trabajos en economía son realmente reproducibles o si resulta muy elevado el coste de la réplica o la reproducción, teniendo en cuenta las escasas posibilidades a efectos de la publicación.

Para concluir merece la pena recordar las palabras de Racine (2017) en una reciente réplica, en la que señala que la ciencia se fortalece cuando podemos verificar un estudio de investigación y progresa cuando podemos extender los estudios. El crecimiento de la ciencia se atrofia si los investigadores no pueden reproducir fácilmente la investigación de los demás.

\section{REFERENCIAS BIBLIOGRÁFICAS}

ARULAMPALAM W., J. HARTOG, T. MACCURDY y J. THEEUWES (1997). "Replication and Re-analysis". Labour Economics, 4, pp. 99-105.

BALL, R. and N. MEDEIROS (2012). "Teaching Integrity in Empirical Research: A Protocol for Documenting Data Analysis and Management". Journal of Economic Education, 43(2), pp. 182-189.

BERNANKE B. (2004). "Editorial Statement”. American Economic Review, 94, pp. 404.

BERRY L., C. COFFMAN, D. HANLEY, R. GIHLEB y A. J. WILSON (2017). "Replication in Microeconomics. Assessing the Rate of Replication in Economics". American Economic Review: Papers \& Proceedings, 107(5), pp. 27-31. 
CAMERER C. F., A. DREBER, E. FORSELL, TECK-HUA HO, J. HUBER, M. JOHANNESSON, M. KIRCHLER, et al. (2016), "Evaluating Replicability of Laboratory Experiments in Economics". Science 351(6280), pp. 1433-36.

CARD D. y A. B. KRUEGER (1994). "Minimum Wages and Employment: A Case Study of the Fast-Food Industry in New Jersey and Pennsylvania". American Economic Review , 84(4), pp. 772-93.

CARD D. y A. B. KRUEGER (2000). "Minimum Wages and Employment: A Case Study of the Fast-Food Industry in New Jersey and Pennsylvania: Reply". American Economic Review, 90(5), pp. 1397-420. [https://doi.org/10.1257/aer.90.5.1397]

CHANG A. y P. LI (2015). "Is Economics Research Replicable? Sixty Published Papers from Thirteen Journals Say "Usually Not". Finance and Economics Discussion Series, September 4, Federal Reserve Board, Washington, D.C.

CLEMENS, M. A. (2017). "The Meaning of Failed Replications: A Review and Proposal". Journal of Economic Surveys, 31, pp. 326-42.

DE JESÚS ROMO V. (2016). “¿Ciencia económica o el arte de hacer economía?. Metodología científica y replicaciones en economía". Investigación Económica, vol. LXXV n 296, abril-junio, pp. 73-110.

DUVENDACK M., R. W. PALMER-JONES y W. R. REED (2015). "Replications in Economics: A Progress Report". Econ Journal Watch, 12(2), pp. 164-191.

DUVENDACK M., R. W. PALMER-JONES y W. R. REED (2017). "Replications and Ethics in Economics: Thirty years after Dewald, Thursby, and Anderson. Why is Meant by "Replication" and Why Does it Encounter Resistance in Economics?". American Economic Review: Papers \& Proceedings, 107(5), pp. 46-51.

HAMERMESH D. S. (1997). "Some Thoughts on Replications and Reviews". Labour Economics, 4, pp. 107-109.

HAMERMESH D. S. (2007). "Viewpoint: Replication in economics". Canadian Journal of Economics, 40(3), pp. 715-33. [https://doi.org/10.1111/j.1365-2966.2007.00428.x]

HAMERMESH D. S. (2007a). "Replication in Economics". NEBER and IZA Discussion Paper, N. ${ }^{\circ}$. 2760. http://www.iza.org/.

HAMERMESH D. S. (2007b). "Viewpoint: Replication in Economics". Canadian Journal of Economics, 40(3), pp. 715-33.

HAMERMESH D. S. (2017). "Replication in Labor Economics: Evidence from Data and What it Suggests". American Economic Review: Papers and Proceeding, 107(5), pp. 37-40.

HANSEN L. P., J. C. HEATON y MASAO OGAKI (1988). "Efficiency Bounds Implied by Multiperiod Conditional Moment Restrictions". Journal of the American Statistical Association, 83, pp. 863-871.

HARRISON T. D. (2003). "Successful Replication of Thornton's (2000) JMCB Article". Indian Journal of Economics and Business, 2, pp. 285.

HÖFFLER J. H. (2014). "Teaching Replication in Quantitative Empirical Economics". Replication Working Paper № 4.

HÖFFLER J. H. (2017). "Improving Transparency in Social Sciences Reserarch". D-Lib Magazine. [https://doi.org/10.1045/march2017-hoeffler]

HOLTZ-EAKIN, D. y T. M SELDEN (1995). "Stoking the fires? $\mathrm{CO}_{2}$ emissions and economic growth". Journal of Political Economy, 57(1), pp. 85-101. 
KANE, E. J. (1984). "Why Journal Editors Should Encourage the Replication of Applied Econometic Research". Quarterly Journal of Business and Economics, 1, pp. 3-8.

LIEBOWITZ S. J., (2017). "A Replication of Four Quasi-Experiments and Three Facts from 'The Effect of File Sharing on Record Sales: An Empirical Analysis' (Journal of Political Economy, 2007)". Economics: The Open-Access, Open-Assessment E-Journal, 11; 2017-13, pp. 1-21. http://dx.doi.org/10.5018/economicsejournal.ja.2017-13.

MCCULLOUGH B. D. y H. D. VINOD (2003). "Verifying the Solution from a Nonlinear Solver: A Case Study". American Economic Review, 93(3), pp. 873-92.

MCCULLOUGH B. D., K. A. MCGEARY y T. D. HARRISON (2006). "Lessons from the JMCB Archive". Journal of Money, Credit and Banking, 38(4), pp. 1093-107.

MERCER R. y R. REED (2015). "A Replication of Economic Development and the Impacts of Natural Disasters (Economics Letters 2007)". Public Finance Review , 43(2), pp.15578.

NEUMARK D. y W. WASCHER (2000). "Minimum Wages and Employment: A Case Study of the Fast-Food Industry in New Jersey and Pennsylvania: Comment". American Economic Review, 90(5), pp. 1362-96.

OBERHOLZER-GEE, F. y STRUMPF K. (2007). "The effect of file sharing on record sales: An empirical analysis". Journal of Political Economy, 115(1), pp. 1-42. http://www.journals.uchicago.edu/doi/abs/10.1086/511995.

PESARAN, HASHEM (2003). "Introducing a replication section". Journal of Applied Econometrics, 18(1), pp. 111.

RACINE J. S. (2017). "Energy, economics, replication \& reproduction". Energy Economics (Available online xxxx), http://dx.doi.org/10.1016/j.eneco.2017.06.027.

SHELDON T. (2017). "Carbon emissions and economic growth: A replication and extension". Energy Economics (Available online xxxx). http://dx.doi.org/10.1016/ j.eneco.2017.03.016

SUKHTANKAR S. (2017). "Replications in Development". American Economic Review: Papers \& Proceedings, 107(5), pp. 32-36. [https://doi.org/10.1257/aer.p20171120]

TOYA H., y M. SKIDMORE (2007). "Economic Development and the Impacts of Natural Disasters". Economics Letters, 94, pp. 20-25.

TOYA H., y M. SKIDMORE (2015). "A Reply to "A Replication of Economic Development and the Impacts of Natural Disasters (Economics Letters 2007)". Public Finance Review, 43(2), pp.179-184. 
